INJE-TP-98-4

\title{
Greybody factor for the BTZ black hole and a 5D black hole
}

\author{
H.W. Lee and Y. S. Myung \\ Department of Physics, Inje University, Kimhae 621-749, Korea
}

\begin{abstract}
We study the 5D black holes in the type IIB superstring theory compactified on $S^{1} \times T^{4}$. Far from horizon, we have flat space-time. Near horizon, we have $A d S_{3}$ (BTZ black hole) $\times S^{3} \times T^{4}$. We calculate the greybody factor of a minimally coupled scalar by replacing the original geometry $\left(M_{5} \times S^{1} \times T^{4}\right)$ by $A d S_{3} \times S^{3} \times T^{4}$. In the low-energy scattering, it turns out that the result agrees with the greybody factor of the 5D black hole (or D1 + D5 branes)in the dilute gas approximation. This confirms that the $A d S$-theory $\left(A d S_{3} \times S^{3} \times T^{4}\right)$ contains the essential information about the bulk 5D black holes.
\end{abstract}




\section{INTRODUCTION}

Recently anti-de Sitter spacetime $(A d S)$ has attracted much interest. It appears that the conjecture relating the string(supergravity) theory on $A d S$ to conformal field theory(CFT) on its boundary may resolve many problems in black hole physics 14 . The 5D black hole correspond to U-dual to BTZ $\times S^{3}$ [5],6]. Sfetsos and Skenderis calculated the entropies of non-extremal 5D black holes by applying Carlip's approach to the BTZ black hole. The BTZ black hole(locally $A d S_{3}$ ) has no curvature singularity [7] and is considered as a prototype for the general CFT/AdS correspondence. This is actually an exact solution of string theory [8] and there is an exact CFT with it on the boundary. Carlip has shown that the physical boundary degrees of freedom account for the Bekenstein-Hawking entropy of the BTZ black hole correctly [9]. Another approach to calculate the entropy of the BTZ black hole was discussed in [10].

Apart from counting the microstates of black holes, the dynamical behavior is also an important issue [11]. This is so because the greybody factor for the black hole arises as a consequence of scattering of a test field off the gravitational potential barrier surrounding the horizon. That is, this is an effect of spacetime curvature. Together with the BekensteinHawking entropy, this seems to be a strong hint of a deep and mysterious connection between curvature and statistical nechanics. It was shown that the s-wave greybody factor for the BTZ black hole has the same form as the 5D black hole in the dilute gas approximation [12.

Here we wish to calculate the greybody factors, using a minimally coupled scalar. The first one is based on the bulk supergravity calculation 13 15 in the effective 5D black hole background. This may be considered as a review of past works. But some discrepancy appears because of the extra dimensions $\left(T^{4}\right)$. Also the D-brane results (effective CFT calculations) are briefly reviewed for comparison. The second is performed by replacing the original geometry $\left(M_{5} \times S^{1} \times T^{4}\right)$ by $A d S_{3} \times S^{3} \times T^{4}$. But we do not require any boundary condition(Dirichlet or Neumann) at the spatial infinity, which is necessary for studying the whole $A d S$. 


\section{GEOMETRY}

The 5D black hole can be obtained as a compactified solution on $S^{1} \times T^{4}$ to the lowenergy effective action of the type IIB theory [16]. This is also represented by the D-brane picture. The near-extremal $5 \mathrm{D}$ black hole is described by the bound states of $Q_{1} 1 \mathrm{D}$-branes and $Q_{5} 5$ D-branes with some momentum flowing along the 1D-brane. Non extremality is achieved by introducing both left and right momenta on the 1D-brane. The supergravity solution in the string frame is given by

$$
\begin{aligned}
d s_{10}^{2}=\left(f_{1} f_{5}\right)^{-1 / 2}[ & \left.-d t^{2}+d x_{5}^{2}+\frac{r_{0}^{2}}{r^{2}}\left(\cosh \sigma d t-\sinh \sigma d x_{5}\right)^{2}+f_{1} d x_{i}^{2}\right] \\
& +\left(f_{1} f_{5}\right)^{1 / 2}\left[h^{-1} d r^{2}+r^{2} d \Omega_{3}^{2}\right]
\end{aligned}
$$

where $f_{1}=1+r_{1}^{2} / r^{2}, f_{5}=1+r_{5}^{2} / r^{2}, h=1-r_{0}^{2} / r^{2}$. The various length scales are given by $r_{1}^{2}=g Q_{1} \alpha^{\prime 3} / V, r_{5}^{2}=g Q_{5} \alpha^{\prime}, r_{0}^{2} \sinh 2 \sigma=2 g^{2} n \alpha^{\prime 4} / R^{2} V$, and $r_{n}^{2}=r_{0}^{2} \sinh ^{2} \sigma$. Here $V\left(\sim \alpha^{\prime 2}\right)$ is the volume of $T^{4}$. The integers $Q_{1}, Q_{5}, n$ are charges for $1 \mathrm{D}, 5 \mathrm{D}$-branes and the total momentum. $g$ is the string coupling and $R$ is the radius of $S^{1}$. The brane configuration lies on a $S^{1} \times T^{4}$ with 1 D-brane along $S^{1}$.

In fact the semiclassical black hole picture and the perturbative D-brane picture are considered to describe the same object in two different regimes. The D-brane picture is expected to describe the semiclassical black hole when $g Q$ is large, whereas the D-brane calculations are performed at weak coupling $(g Q \ll 1)$. For extremal BPS states there are well-known non-renormalization theorems which state that the degeneracy of states do not change as $g Q$ increases. However, for non-BPS states including the dilute gas limit, there are no such theorems. One wishes to resolve this puzzle with a new idea. For this purpose, one may introduce the decoupling limit which should be taken to suppress closed string loop $\operatorname{corrections}(g \rightarrow 0)$ and higher-order terms in the $\alpha^{\prime}$-expansion $\left(\alpha^{\prime} \rightarrow 0\right)$ [1]. This is given by

$$
g \rightarrow 0, \alpha^{\prime} \rightarrow 0 ; g Q_{1}, g Q_{5} \gg 1 \text { with } r_{1}, r_{5}, r_{n} \text { fixed. }
$$


Actually (2) corresponds to the dilute gas limit of $r_{0}, r_{n} \ll r_{1}, r_{5}$. The size of this black hole is controlled by $g Q_{1}$ and $g Q_{5}$. Its thermodynamic quantities are given by

$$
\begin{aligned}
M & =\frac{\pi}{4 G_{N}^{5 D}}\left[r_{1}^{2}+r_{5}^{2}+\frac{1}{2} r_{0}^{2} \cosh 2 \sigma\right], \\
S & =\frac{\mathcal{A}_{H}^{5 D}}{4 G_{N}^{5 D}}=\frac{\pi^{2} r_{1} r_{5} r_{0} \cosh \sigma}{2 G_{N}^{5 D}} \\
T_{H} & =\left(\frac{2 \pi}{r_{0}} r_{1} r_{5} \cosh \sigma\right)^{-1}
\end{aligned}
$$

with the 5D Newton constant $\left(G_{N}^{5 D}\right)$. The above energy and entropy are essentially those of a gas of massless 1D particles. In this case the temperatures for left and right moving string modes are defined by

$$
T_{L}=\frac{1}{2 \pi}\left(\frac{r_{0}}{r_{1} r_{5}}\right) e^{\sigma}, \quad T_{R}=\frac{1}{2 \pi}\left(\frac{r_{0}}{r_{1} r_{5}}\right) e^{-\sigma} .
$$

In the decoupling limit(near horizon, $r \simeq r_{0}$ ) the metric (1) leads to

$$
\begin{aligned}
d s_{10}^{2}=\frac{r^{2}}{R^{2}}\left(-d t^{2}\right. & \left.+d x_{5}^{2}\right)+\frac{r_{0}^{2}}{R^{2}}\left(\cosh \sigma d t-\sinh \sigma d x_{5}\right)^{2} \\
& +\frac{R^{2}}{r^{2}}\left(1-\frac{r_{0}^{2}}{r^{2}}\right)^{-1} d r^{2}+R^{2} d \Omega_{3}^{2}+\frac{r_{1}}{r_{5}} d x_{i}^{2}
\end{aligned}
$$

with $R^{2}=r_{1} r_{5}$. Using the coordinate transformation $\rho^{2}=r^{2}+r_{0}^{2} \sinh ^{2} \sigma$, one finds the well-known metric [5,6, [17]

$$
d s_{10}^{2}=d s_{B T Z}^{2}+R^{2} d \Omega_{3}^{2}+\frac{r_{1}^{2}}{R^{2}} d x_{i}^{2},
$$

where the BTZ black hole space-time is given by [7]

$$
d s_{B T Z}^{2}=-\frac{\left(\rho^{2}-\rho_{+}^{2}\right)\left(\rho^{2}-\rho_{-}^{2}\right)}{\rho^{2} R^{2}} d t^{2}+\rho^{2}\left(d \varphi-\frac{J}{2 \rho^{2}} d t\right)^{2}+\frac{\rho^{2} R^{2}}{\left(\rho^{2}-\rho_{+}^{2}\right)\left(\rho^{2}-\rho_{-}^{2}\right)} d \rho^{2} .
$$

Here $M=\left(\rho_{+}^{2}+\rho_{-}^{2}\right) / R^{2}, J=2 \rho_{+} \rho_{-} / R$ are the mass and angular momentum of the BTZ black hole, and $\rho_{+}=r_{0} \cosh \sigma, \rho_{-}=r_{0} \sinh \sigma, \varphi=x_{5} / R$. In this case, the relevant thermodynamic quantities(Hawking temperature, area of horizon, angular velocity at horizon, left/right temperatures) are given by 


$$
\begin{aligned}
& T_{H}^{B T Z}=\frac{\rho_{+}^{2}-\rho_{-}^{2}}{2 \pi R^{2} \rho_{+}}=T_{H}, \\
& \mathcal{A}_{H}^{B T Z}=2 \pi \rho_{+}, \quad \Omega_{H}=\frac{J}{2 \rho_{+}^{2}}, \\
& \frac{1}{T_{L / R}^{B T Z}}=\frac{1}{T_{H}^{B T Z}}\left(1 \pm \frac{\rho_{+}}{\rho_{-}}\right)=\frac{1}{T_{L / R}} .
\end{aligned}
$$

\section{SUPERGRAVITY CALCULATION : SCATTERING IN 5D BLACK HOLE}

We consider the scattering of a free scalar in the whole region of 5D black hole. This means that our calculation corresponds to the bulk supergravity calculation. In the Einstein frame, $d s_{10}^{2}$ can be reduced to the 5D black hole and additional Kaluza-Klein moduli $(\chi, \nu)$ as [13]

$$
\left(d s_{10}^{2}\right)_{E}=e^{-\phi / 2} d s_{10}^{2}=e^{2 \chi} \sum_{i=6}^{9} d x_{i}^{2}+e^{2 \nu}\left(d x_{5}+A_{\mu} d x^{\mu}\right)^{2}+e^{-2(4 \chi+\nu) / 3} d s_{5 D}^{2}
$$

with $\mu=0,1,2,3,4$. Here $e^{2 \chi}=f_{1}^{1 / 4} f_{5}^{-1 / 4}, e^{2 \nu}=f_{1}^{-3 / 4} f_{5}^{-1 / 4} f_{n}$, the Kaulza-Klein gauge potential $A_{0}=-r_{0}^{2} \sinh 2 \sigma / 2\left(r^{2}+r_{n}^{2}\right)$, and the $5 \mathrm{D}$ black hole space-time is given by

$$
d s_{5 D}^{2}=-f^{-2 / 3} h d t^{2}+f^{1 / 3}\left[h^{-1} d r^{2}+r^{2} d \Omega_{3}^{2}\right]
$$

with $f=f_{1} f_{5} f_{n}$ with $f_{n}=1+r_{n}^{2} / r^{2}$.

First let us consider the wave equation in the ten-dimensional background (9),

$$
\square_{10} \Phi=0
$$

Considering the symmetries in (9), $\Phi$ can be seperated as

$$
\Phi=e^{-i \omega t} e^{i K_{5} x_{5}} e^{i K_{i} x^{i}} Y_{l}\left(\theta_{1}, \theta_{2}, \theta_{3}\right) \phi(r)
$$

For simplicity, we take $K_{5}=0, r_{1} \simeq r_{5}$. Further we set $A_{0}=0$, because it approaches to zero in the dilute gas limit. Then (11) leads to a new 5D wave equation for a free scalar

$$
\left[\frac{d^{2}}{d r^{2}}+\left(\frac{h^{\prime}}{h}+\frac{3}{r}\right) \frac{d}{d r}\right] \phi+\frac{\omega^{2} f}{h^{2}} \phi-\frac{l(l+2)}{h r^{2}} \phi-\frac{K^{2} f_{5}^{2}}{h} \phi=0 .
$$


For $l=K_{i}=0$ case, Eq.(13) corresponds to the wave equation of Ref. [11] and we recover that of Refs. 14,15] for $K_{i}=0$. We confine ourselves to low energies satisfying $r_{1} \omega, r_{5} \omega<1$. As usual we cannot find a solution to (13) analytically and thus we devide the space into a far region $\left(r \gg r_{1}, r_{5}\right)$ and a near region $\left(r \ll r_{1}, r_{5}\right)$. We can match the solution in the overlapping region $\left(\omega r_{0} \ll \omega r \ll 1\right)$ because $\omega r_{0} \ll \omega r_{1}, \omega r_{5}<1$ in the dilute gas limit $13,15,18$.

In the far region we write $\phi=\tilde{\phi} / r$, and then the equation for $\phi$ leads to

$$
\frac{d^{2} \tilde{\phi}}{d u^{2}}+\frac{1}{u} \frac{d \tilde{\phi}}{d u}+\left[1-\frac{\nu^{2}}{u^{2}}\right] \tilde{\phi}=0
$$

with $u=\omega^{\prime} r$ and $\nu^{2}=(l+1)^{2}-\left(r_{1}^{2}+r_{n}^{2}\right) K^{2}-\left(\rho_{1}^{2}+\rho_{5}^{2}+\rho_{n}^{2}\right)$. Here $\omega^{\prime}=\sqrt{\omega^{2}-K^{2}}, \rho_{i}=\omega^{\prime} r_{i}$ and $K_{i} r_{i}<\rho_{i}<\omega r_{i}$. The solutions are given by the Bessel function when $\nu$ is not an integer,

$$
\phi_{f}=\frac{1}{u}\left[\alpha J_{\nu}(u)+\beta J_{-\nu}(u)\right],
$$

where $\alpha, \beta$ are unknown constants. From the large- $u$ behavior, one finds the incoming flux

$$
\begin{aligned}
\mathcal{F}_{\text {in }}(\infty) & =\left.\frac{2 \pi}{i}\left[\phi_{f}^{\text {in } *} r^{3} \partial_{r} \phi_{f}^{\text {in }}-\phi_{f}^{\text {in }} r^{3} \partial_{r} \phi_{f}^{\text {in } *}\right]\right|_{r=\infty} \\
& =-\frac{2}{\omega^{\prime 2}}\left|\alpha e^{i(\nu+1 / 2) \pi / 2}+\beta e^{i(-\nu+1 / 2) \pi / 2}\right|^{2},
\end{aligned}
$$

where $\phi_{f}^{\text {in }}$ is given by

$$
\phi_{f}^{\text {in }}=\sqrt{\frac{1}{2 \pi}} \frac{e^{-i u}}{u^{3 / 2}}\left\{\alpha e^{i(\nu+1 / 2) \pi / 2}+\beta e^{i(-\nu+1 / 2) \pi / 2}\right\} .
$$

The small- $u$ behavior of $\phi_{f}$ is

$$
\phi_{\mathrm{f} \rightarrow \text { inter }}=\frac{1}{u}\left[\alpha\left(\frac{u}{2}\right)^{\nu} \frac{1}{\Gamma(\nu+1)}+\beta\left(\frac{u}{2}\right)^{-\nu} \frac{1}{\Gamma(-\nu+1)}\right] .
$$

Here we note that $\phi_{\mathrm{f} \rightarrow \text { inter }}$ has not any pole because $\nu$ is not integer.

Now we wish to find the solution in the near region. Using $h=1-r_{0}^{2} / r^{2}$, the wave equation (13) can be rewritten as

$$
h(1-h) \frac{d^{2} \phi}{d h^{2}}+(1-h) \frac{d \phi}{d h}+\left\{-C+\frac{C+D+E}{h}+\frac{E}{1-h}\right\} \phi=0,
$$


where

$$
\begin{aligned}
& C=\left(\frac{\omega r_{1} r_{5} r_{n}}{2 r_{0}^{2}}\right)^{2} \\
& D=\frac{l(l+2)}{4}+\frac{\omega^{2}}{4 r_{0}^{2}}\left[r_{1}^{2} r_{5}^{2}+r_{n}^{2}\left(r_{1}^{2}+r_{5}^{2}\right)\right]+\frac{K^{2} r_{5}^{2}}{4} \\
& E=-\frac{l(l+2)}{4}+\frac{\omega^{2}\left(r_{1}^{2}+r_{5}^{2}+r_{n}^{2}\right)}{4}-\frac{K^{2}\left(r_{5}^{2}-r_{0}^{2}\right)}{4}
\end{aligned}
$$

Hereafter we set $K^{2} r_{0}^{2}, K^{2} r_{n}^{2} \simeq 0$. With an unknown constant $A$, we find the ingoing mode at the horizon

$$
\phi_{n}^{\text {in }}=A h^{-i q}(1-h)^{(1-\nu) / 2} F(a, b, c ; h),
$$

where

$$
\begin{aligned}
& a=\frac{1-\nu}{2}-i q+i \sqrt{C} \\
& b=\frac{1-\nu}{2}-i q-i \sqrt{C} \\
& c=1-2 i q \\
& q=\frac{\omega}{4 \pi T_{H}} \sqrt{\left(1+\frac{r_{0}^{2}}{r_{1}^{2}}+\frac{r_{0}^{2}}{r_{5}^{2}}\right)+4 \pi^{2} r_{n}^{2} T_{H}^{2}} .
\end{aligned}
$$

The large- $r$ behavior $(h \rightarrow 1)$ follows from the $(h \rightarrow 1-h)$ transformation law for hypergeometric functions as 19

$$
\begin{aligned}
\phi_{n \rightarrow f}= & \frac{A \Gamma(1-2 i q) \Gamma(\nu)}{\Gamma\left(\frac{1+\nu}{2}-i q-i \sqrt{C}\right) \Gamma\left(\frac{1+\nu}{2}-i q+i \sqrt{C}\right)} u^{\nu-1} \\
& +\frac{A \Gamma(1-2 i q) \Gamma(-\nu)}{\Gamma\left(\frac{1-\nu}{2}-i q-i \sqrt{C}\right) \Gamma\left(\frac{1-\nu}{2}-i q+i \sqrt{C}\right)} u^{-\nu-1}
\end{aligned}
$$

Matching (18) with (21) leads to

$$
\begin{aligned}
& \alpha=A u_{0} \frac{2^{\nu} \Gamma(\nu+1) \Gamma(\nu) \Gamma(1-2 i q) u_{0}^{-\nu}}{\Gamma\left(\frac{1+\nu}{2}-i q-i \sqrt{C}\right) \Gamma\left(\frac{1+\nu}{2}-i q+i \sqrt{C}\right)}, \\
& \beta=A u_{0} \frac{\Gamma(-\nu+1) \Gamma(-\nu) \Gamma(1-2 i q) u_{0}^{\nu}}{2^{\nu} \Gamma\left(\frac{1-\nu}{2}-i q-i \sqrt{C}\right) \Gamma\left(\frac{1-\nu}{2}-i q+i \sqrt{C}\right)} .
\end{aligned}
$$

Since $u_{0}\left(=\omega^{\prime} r_{0}\right) \ll 1$, one finds $\beta \ll \alpha$.

Further the flux across the horizon is calculated as 


$$
\mathcal{F}_{\text {in }}(0)=-8 \pi r_{0}^{2} q|A|^{2}
$$

Then the absoprtion coefficient is given by

$$
\mathcal{A}=\frac{\mathcal{F}_{\text {in }}(0)}{\mathcal{F}_{\text {in }}(\infty)} \simeq 4 \pi u_{0}^{2} q\left|\frac{A}{\alpha}\right|^{2}
$$

The new absorption cross section takes the from

$$
\begin{aligned}
\sigma_{\mathrm{abs}}^{5 D}=(l+1)^{2} \frac{4 \pi}{\omega^{3}} \mathcal{A}= & \left(\frac{\omega^{\prime}}{\omega}\right)^{2} \mathcal{A}_{H}^{5 D}\left(\omega r_{0}\right)^{2(\nu-1)}(l+1)^{2}\left(1+\frac{r_{0}^{2}}{r_{1}^{2}}+\frac{r_{0}^{2}}{r_{5}^{2}}+4 \pi^{2} r_{n}^{2} T_{H}^{2}\right)^{1 / 2} \\
& \times\left|\frac{2^{-\nu+1}}{\Gamma(\nu) \Gamma(\nu+1)}\right|^{2}\left|\frac{\Gamma\left(\frac{1+\nu}{2}-i q-i \sqrt{C}\right) \Gamma\left(\frac{1+\nu}{2}-i q+i \sqrt{C}\right)}{\Gamma(1-2 i q)}\right|^{2} .
\end{aligned}
$$

The decay rate for $K \simeq 0, q \simeq \omega / 4 \pi T_{H}, \nu \simeq l+1$, is given by

$$
\Gamma_{5 D}=\frac{\sigma_{\mathrm{abs}}^{5 D}}{e^{\omega / T_{H}}-1}=\frac{\pi r_{0}^{2 l+2} \omega^{2 l-1}(l+1)^{2} e^{-\omega / 2 T_{H}}}{|\Gamma(l+1) \Gamma(l+2)|^{2}}\left|\Gamma\left(1+\frac{l}{2}+i \frac{\omega}{2 \pi T_{L}}\right) \Gamma\left(1+\frac{l}{2}+i \frac{\omega}{2 \pi T_{R}}\right)\right|^{2} .
$$

We now compare this decay rate with the CFT prediction. In the effective string picture(Dbrane picture) such decays are described by a coupling of the space-time scalar field to an operator with dimension 1 in the conformal field theory, both in the left- and right-moving sector. A calculation showed that the decay rate is given by [14]

$$
\tilde{\Gamma}_{5 D}=\frac{\Gamma_{5 D}}{(l+1)^{2}}
$$

Hence the CFT prediction $\left(\tilde{\Gamma}_{5 D}\right)$ and the semiclassical decay rate $\left(\Gamma_{5 D}\right)$ agree at least when $l=0$. Upto the first-order approximation $\left(\omega^{\prime} \simeq \omega\left(1-\frac{K^{2}}{2 \omega^{2}}\right), q \simeq \omega / 4 \pi T_{H}, \nu \simeq l+1-\left(K^{2}+\right.\right.$ $\left.\left.2 \omega^{2}\right) r_{5}^{2} / 2(l+1)\right)$, we obtain the absorption cross section for $M_{5} \times S^{1}$

$$
\begin{aligned}
\left(\sigma_{\mathrm{abs}}^{6 D}\right)^{1}=(1 & \left.-\frac{K^{2}}{2 \omega^{2}}\right)^{2} \mathcal{A}_{H}^{6 D}\left(\omega\left(1-\frac{\left(K^{2}+2 \omega^{2}\right)}{2 \omega^{2}}\right) r_{0}\right)^{2 l-\left(K^{2}+2 \omega^{2}\right) r_{5}^{2} /(l+1)}(l+1)^{2} \\
& \times\left|\frac{2^{-l}}{\Gamma\left(l+1-\frac{\left(K^{2}+2 \omega^{2}\right) r_{5}^{2}}{2(l+1)}\right) \Gamma\left(l+2-\frac{\left(K^{2}+2 \omega^{2}\right) r_{5}^{2}}{2(l+1)}\right)}\right|^{2} \\
& \times\left|\frac{\Gamma\left(1+\frac{l}{2}-\frac{\left(K^{2}+2 \omega^{2}\right) r_{5}^{2}}{4(l+1)}-i \frac{\omega}{4 \pi T_{L}}\right) \Gamma\left(1+\frac{l}{2}-\frac{\left(K^{2}+2 \omega 62\right) r_{5}^{2}}{4(l+1)}-i \frac{\omega}{4 \pi T_{R}}\right)}{\Gamma\left(1-i \frac{\omega}{2 \pi T_{H}}\right)}\right|^{2} .
\end{aligned}
$$

Here we used the dilute gas approximation $\left(r_{1} \simeq r_{5} \simeq R \gg r_{0}\right)$ and $\mathcal{A}_{H}^{6 D}=\mathcal{A}_{H}^{5 D} \times 2 \pi R=$ $4 \pi^{3} r_{n} R^{3}$. In this case one finds the relation $\left(\mathcal{A}_{H}^{6 D} T_{H}=2 \pi^{2} r_{0}^{2} R\right)$. This is actually the same result with the geometry such as $A d S_{3} \times S^{3} \times T^{4}$ near horizon, and flat space-time at spatial infinity. 


\section{IV. $A D S$-CALCULATION : SCATTERING IN $A D S_{3} \times S^{3} \times T^{4}$}

Here we consider the geometry such as the $A d S_{3} \times S^{3} \times T^{4}$ near horizon, and the flat space-time at spatial infinity. A ten-dimensional minimally coupled scalar in the background of (6) satisfies the wave equation

$$
\square_{10} \Psi=0 .
$$

$\Psi$ can be decomposed into

$$
\Psi=e^{-i \omega t} e^{i m \varphi} e^{i K_{i} x^{i}} Y_{l}\left(\theta_{1}, \theta_{2}, \theta_{3}\right) \psi(\rho)
$$

Then (30) leads to

$$
\nabla_{B T Z}^{2} \psi(\rho)+\frac{\mu}{R^{2}} \psi(\rho)=0
$$

with $\mu=-l(l+2)-K^{2} r_{5}^{2}$. Since it is hard to find a solution to (32) directly, we use the matching procedure [20]. The space is devided into two regions: the near region $\left(\rho \sim \rho_{+}\right)$ and far region $(\rho \rightarrow \infty)$. In the far region (32) becomes

$$
\frac{d^{2} \psi_{\infty}}{d x^{2}}+\frac{3}{x} \frac{d \psi_{\infty}}{d x}+\frac{\mu}{x^{2}} \psi_{\infty}=0
$$

with a dimensionless quantity $x=\rho / R$. Here one finds the far-region solution

$$
\psi_{\infty}(x)=\frac{1}{x}\left[\tilde{\alpha} x^{\sqrt{1-\mu}}+\tilde{\beta} x^{-\sqrt{1-\mu}}\right]
$$

The ingoing flux at infinity is calculated as

$$
\mathcal{F}_{\text {in }}(\infty)=-2 \pi \sqrt{1-\mu}|\tilde{\alpha}-i \tilde{\beta}|^{2}
$$

In order to obtain the near-region behavior, we introduce the variable $z=\left(\rho^{2}-\rho_{+}^{2}\right) /\left(\rho^{2}-\right.$ $\left.\rho_{-}^{2}\right)=\left(x^{2}-x_{+}^{2}\right) /\left(x^{2}-x_{-}^{2}\right)$. Then (32) becomes

$$
z(1-z) \frac{d^{2} \psi}{d z^{2}}+(1-z) \frac{d \psi}{d z}+\left(\frac{A_{1}}{z}+\frac{\mu / 4}{1-z}+B_{1}\right) \psi=0,
$$

where 


$$
A_{1}=\left(\frac{\omega-m \Omega_{H}}{4 \pi T_{H}^{B T Z}}\right)^{2}, B_{1}=-\frac{\rho_{-}^{2}}{\rho_{+}^{2}}\left(\frac{\omega-m \Omega_{H} \rho_{+}^{2} / \rho_{-}^{2}}{4 \pi T_{H}^{B T Z}}\right)^{2} .
$$

The ingoing solution for (36) near horizon is given by the hypergeometric function

$$
\psi(z)=C_{1} z^{-i \sqrt{A_{1}}}(1-z)^{(1-\sqrt{1-\mu}) / 2} F(a, b, c ; z)
$$

where

$$
\begin{aligned}
& a=\sqrt{B_{1}}-i \sqrt{A_{1}}+(1-\sqrt{1-\mu}) / 2, \\
& b=-\sqrt{B_{1}}-i \sqrt{A_{1}}+(1-\sqrt{1-\mu}) / 2, \\
& c=1-2 i \sqrt{A_{1}} .
\end{aligned}
$$

The corresponding flux is given by

$$
\mathcal{F}_{\text {in }}(0)=-8 \pi \sqrt{A_{1}}\left(x_{+}^{2}-x_{-}^{2}\right)\left|C_{1}\right|^{2} .
$$

The absorption coefficient is calculated as

$$
\mathcal{A}=\frac{\mathcal{F}_{\text {in }}(0)}{\mathcal{F}_{\text {in }}(\infty)}=\frac{4 \sqrt{A_{1}}\left(x_{+}^{2}-x_{-}^{2}\right)}{\sqrt{1-s}} \frac{\left|C_{1}\right|^{2}}{|\tilde{\alpha}-i \tilde{\beta}|^{2}} .
$$

To obtain $\tilde{\alpha}$ and $\tilde{\beta}$, we use the matching procedure. First, we have to know the $z \rightarrow$ 1 behavior of (38). This can be obtained by the transformation rule $(z \rightarrow 1-z)$ for hypergeometric function [19]. It takes the form

$$
\psi_{n \rightarrow f}(z)=\frac{1}{x}\left[C_{1} E_{1}\left(x_{+}^{2}-x_{-}^{2}\right)^{(1-\sqrt{1-\mu}) / 2} x^{\sqrt{1-\mu}}+C_{1} E_{2}\left(x_{+}^{2}-x_{-}^{2}\right)^{(1+\sqrt{1-\mu}) / 2} x^{-\sqrt{1-\mu}}\right]
$$

where

$$
\begin{aligned}
& E_{1}=\frac{\Gamma\left(1-2 i \sqrt{A_{1}}\right) \Gamma(\sqrt{1-\mu})}{\Gamma\left(\frac{1+\sqrt{1-\mu}}{2}-\sqrt{B_{1}}-i \sqrt{A_{1}}\right) \Gamma\left(\frac{1+\sqrt{1-\mu}}{2}+\sqrt{B_{1}}-i \sqrt{A_{1}}\right)}, \\
& E_{2}=\frac{\Gamma\left(1-2 i \sqrt{A_{1}}\right) \Gamma(-\sqrt{1-\mu})}{\Gamma\left(\frac{1-\sqrt{1-\mu}}{2}-\sqrt{B_{1}}-i \sqrt{A_{1}}\right) \Gamma\left(\frac{1-\sqrt{1-\mu}}{2}+\sqrt{B_{1}}-i \sqrt{A_{1}}\right)},
\end{aligned}
$$

In matching (34) with (41), we find

$$
\tilde{\alpha}=C_{1} E_{1}\left(\frac{r_{0}}{R}\right)^{1-\sqrt{1-\mu}}, \quad \tilde{\beta}=C_{1} E_{2}\left(\frac{r_{0}}{R}\right)^{1+\sqrt{1-\mu}} .
$$


Considering $R\left(=\sqrt{r_{1} r_{5}}\right) \gg r_{0}$, one finds $\tilde{\beta} \ll \tilde{\alpha}$. Then the absorption coefficient is approximately given by

$$
\mathcal{A} \simeq \frac{4 \sqrt{A_{1}}\left(x_{+}^{2}-x_{-}^{2}\right)}{\sqrt{1-\mu}}\left(\frac{r_{0}}{R}\right)^{2(\sqrt{1-\mu}-1)} \frac{1}{\left|E_{1}\right|^{2}}
$$

The absorption cross section for $A d S_{3} \times S^{3}$ with $m=0$ leads to

$$
\begin{aligned}
\tilde{\sigma}_{\mathrm{abs}}^{6 D}=c \frac{\mathcal{A}}{\omega}= & c \frac{\mathcal{A}_{H}^{B T Z}}{\pi}\left(\frac{r_{0}}{R}\right)^{2(\sqrt{1-\mu}-1)} \frac{1}{\Gamma(1+\sqrt{1-\mu}) \Gamma(\sqrt{1-\mu})} \\
& \times\left|\frac{\Gamma\left(\frac{1+\sqrt{1-\mu}}{2}-i \frac{\omega}{4 \pi T_{L}^{B T Z}}\right) \Gamma\left(\frac{1+\sqrt{1-\mu}}{2}-i \frac{\omega}{4 \pi T_{R}^{B T Z}}\right)}{\Gamma\left(1-i \frac{\omega}{2 \pi T_{H}^{B T Z}}\right)}\right|^{2} .
\end{aligned}
$$

Here $c$ can be determined by requiring that $\lim _{\omega \rightarrow 0, s \rightarrow 0} \tilde{\sigma}_{\text {abs }}^{6 D}=\tilde{\mathcal{A}}_{H}^{6 D}=\mathcal{A}_{H}^{\mathrm{BTZ}} \times 2 \pi^{2} R^{3}$. In this case the non-compact space-time is three dimensions, so that the conversion factor from $\mathcal{A}$ to $\sigma$ is $1 / \omega$. Also one finds the relation : $\tilde{\mathcal{A}}_{H}^{6 D}=\mathcal{A}_{H}^{6 D}$. Upto the first-order approximation $\left(\sqrt{1-\mu} \simeq l+1+\frac{r_{5}^{2} K^{2}}{2(l+1)}\right)$, (44) leads to

$$
\begin{aligned}
\left(\tilde{\sigma}_{\text {abs }}^{6 D}\right)^{1}= & \tilde{\mathcal{A}}_{H}^{6 D}\left(\frac{r_{0}}{R}\right)^{2 l+\frac{r_{5}^{2} K^{2}}{l+1}} \frac{1}{\Gamma\left(l+1+\frac{r_{5}^{2} K^{2}}{2(l+1)}\right) \Gamma\left(l+2+\frac{r_{5}^{2} K^{2}}{2(l+1)}\right)} \\
& \times\left|\frac{\Gamma\left(1+\frac{l}{2}+\frac{r_{5}^{2} K^{2}}{4(l+1)}-i \frac{\omega}{4 \pi T_{L}^{B T Z}}\right) \Gamma\left(1+\frac{l}{2}+\frac{r_{5}^{2} K^{2}}{4(l+1)}-i \frac{\omega}{4 \pi T_{R}^{B T Z}}\right)}{\Gamma\left(1-i \frac{\omega}{2 \pi T_{H}^{B T Z}}\right)}\right|^{2} .
\end{aligned}
$$

\section{DISCUSSION}

We investigate the dynamical behavior of the 5D black holes with a minimally coupled scalar field. Apart from counting the microstates of black holes, the dynamical behavior is also an important issue. This is so because the greybody factor(absorption cross section) for the black hole arises as a consequence of scattering of a free scalar off the potential surrounding the horizon. That is, this is an effect of spacetime curvature.

First we obtain the greybody factor for 5D black hole by using the supergravity calculation. Second, we calculate the absorption cross section of a minimally coupled scalar by replacing the $5 \mathrm{D}$ black hole by $A d S_{3} \times S^{3} \times T^{4}$. Here we do not require any boundary condition at infinity. Actually we use the matching of $A d S$ region to obtain $\tilde{\sigma}_{\text {abs }}^{6 D}$. In this sense 
our case is asymptotically anti-de Sitter space but not asymptotically flat space. On the other hand, in studying the $A d S_{3}$, one usually requires the boundary conditions at spatial infinity [3,20 22]. This is so because $A d S_{3}$ is not globally hyperbolic. The spatial infinity of the BTZ black hole is timelike, so the information may enter or exit from the bounadry at infinity. Thus one imposes boundary condition at infinity to obtain the sensible results.

Birmingham et al. showed that the s-wave greybody factor for the BTZ black hole $\left(A d S_{3}\right)$ has the same form as the one for $5 \mathrm{D}$ black hole $\left(M_{5}\right)$ in the dilute gas approximation [12]. In the low-energy approximation, we find the same absorption cross section for both two $\operatorname{cases}\left(M_{5} \times S^{1} \times T^{4}, A d S_{3} \times S^{3} \times T^{4}\right)$ as

$$
\left(\sigma_{\mathrm{abs}}^{6 D}\right)_{l=0, K=0}=\left(\tilde{\sigma}_{\mathrm{abs}}^{6 D}\right)_{l=0, K=0} \simeq \mathcal{A}_{H}^{6 D}\left|\frac{\Gamma\left(1-i \frac{\omega}{4 \pi T_{L}}\right) \Gamma\left(1-i \frac{\omega}{4 \pi T_{R}}\right)}{\Gamma\left(1-i \frac{\omega}{2 \pi T_{H}}\right)}\right|^{2} .
$$

This means that the relevant information of $5 \mathrm{D}$ black hole are encoded in the $A d S$-theory [10,17,23. Actually the greybody factor is determined by the potential surrounding the 5D black hole. If we introduce a tortoise coordinate $r_{*}=r+r_{0} \ln \left|\left(r-r_{0}\right) /\left(r+r_{0}\right)\right| / 2$ [11], Eq.(13) can be rewritten as

$$
\left[-\frac{d^{2}}{d r_{*}^{2}}+V_{\omega}\left(r_{*}\right)\right] \phi=0
$$

where the $l=0$ potential $V_{\omega}$ is given by

$$
V_{\omega}\left(r_{*}\right)=-\omega^{2} f+K^{2} f_{5} h+\frac{3 h}{4 r^{2}}\left(1+\frac{3 r_{0}^{2}}{r^{2}}\right) .
$$

In the decoupling limit, (48) reduces to

$$
V_{\omega}^{d-l}\left(r_{*}\right)=-\omega^{2} \frac{R^{4}}{r^{4}}\left(1+\frac{r_{n}^{2}}{r^{2}}\right)+K^{2} \frac{R^{2}}{r^{2}} h+\frac{3 h}{4 r^{2}}\left(1+\frac{3 r_{0}^{2}}{r^{2}}\right),
$$

which corresponds actually to the potential for the $A d S_{3} \times S^{3} \times T^{4}$ near horizon. In the lowenergy $\operatorname{limit}(\omega \rightarrow 0, K \rightarrow 0)$, two potentials $\left(V_{\omega}\right.$ and $\left.V_{\omega}^{d-l}\right)$ take the same form. Hence it is clear that the absorption cross section of $5 \mathrm{D}$ black hole is equal to that of the $A d S_{3} \times S^{3} \times T^{4}$ with asymptotically flat space.

In conclusion, we calculate the absorption cross section for a minimally coupled scalar in the $M_{5} \times S^{1} \times T^{4}$ and $A d S_{3} \times S^{3} \times T^{4}$ supergravities. We note that the holography is related 
to theories of different domensionality [1] 3]. Here our working dimension is the same for two theories. In the low-energy limit of $\omega \rightarrow 0$, two cross sections are same. This shows a signal for holographic duality in the supergravity side.

Finally we comment on the greybody factors. The calculations of greybody factor for a free scalar in $M_{5}(5 \mathrm{D}$ black hole background) have already appeared in the literature 11, 14, 15. In our calculation, some discrepancy appears because of the extra dimensions $\left(T^{4}\right)$. For the $A d S_{3}$-calculation, Birmingham et al. first calculated the cross section for a free scalar [12. Although this is correct, the calculation procedure is incomplete. This is so because they did not consider the boundary condition at infinity seriously. One cannot obtain the cross section for a free scalar in the $A d S_{3}$ by requiring the Dirichlet or Neumann boundary condition at spatial infinity. Our calculation for $A d S_{3} \times S^{3} \times T^{4}$ is done with asymptotically $A d S_{3}$. However we do not need to consider the boundary condition. Actually this is based on the non-normalizable mode ( $\tilde{\alpha}$ - dependent term in (34)) without the boundary condition [3:24]. Even in the asymptotically $A d S_{3}$, it seems that one can calculate the absorption cross section in the $A d S_{3}$ by following our procedure.

\section{ACKNOWLEDGEMENT}

This work was supported in part by the Basic Science Research Institute Program, Minstry of Education, Project NOs. BSRI-97-2413 and BSRI-97-2441. 


\section{REFERENCES}

[1] J. Maldacena, hep-th/9711200.

[2] S.S. Gubser, I.R. Klebanov and A.M. Polyakov, hep-th/9802109.

[3] E. Witten, hep-th/9802150.

[4] G.T. Horowitz and H. Oguri, hep-th/9802116; E. Witten, hep-th/9803131; G.T. Horowitz and S. Ross, hep-th/9803085.

[5] S. Hyun, hep-th/9704005.

[6] K. Sfetsos and K. Skenderis, hep-th/9711138.

[7] M. Banados, C. Teitelboim and A. Zanelli, Phys. Rev. Lett. 69 (1992)1849; S. Carlip, Class. Quant. Grav. 12 (1995)2853.

[8] G. Horowitz and D. Welch, Phys. Rev. Lett. 71 (1993)328; N. Kaloper, Phys. Rev. D48 (1993)2598; A. Ali and A. Kumar, Mod. Phys. Lett. A8 (1993)2045.

[9] S. Carlip, Phys. Rev. D51 (1995)632; D55 (1997)878.

[10] A. Strominger, hep-th/9712251; D. Birmingham, I. Sachs ans S. Sen, hep-th/9801019; N. Kaloper, hep-th/9804062.

[11] A. Dhar, G. Mandal and S. Wadia, Phys. Lett. B388 (1996)51; S. Das, G. Gibbons and S. Mathur, Phys. Rev. Lett. 78 (1997)417.

[12] D.B. Birmingham, I. Sachs and S. Sen, Phys. Lett. B413 (1997)281.

[13] F. Dowker, D. Kastor and J. Traschen, hep-th/9702109.

[14] S.D. Mathur, hep-th/9704050; S.S. Gubser, hep-th/9704195.

[15] J. Maldacena and A. Strominger, Phys. Rev. D56 (1997) 4975.

[16] G.T. Horowitz, J.M. Maldacena and A. Strominger, Phys. Lett. B383 (1996)151; J. 
Maldacena, hep-th/9607235.

[17] S.P. de Alwis, hep-th/9804019.

[18] C. Callan, S. Gubser, I.G. Klebanov and A. Tseytlin, Nucl. Phys. B489 (1997)65; M. Krasnitz and I.G. Klebanov, hep-th/9703216; B. Kol and A. Rajaraman, Phys. Rev. D56 (1997)983; H.W. Lee, Y.S. Myung and J.Y. Kim, Phys. Lett. B410 (1997)6; hepth/9708099.

[19] M. Abramowitz and I. Stegun, Handbook of Mathematical Functions (Academic Press, New York, 1966) p.559.

[20] H.W. Lee, N.J. Kim, and Y.S. Myung, hep-th/9803080; hep-th/9803227.

[21] G. Lifschytz and M. Oritz, Phys. Rev. D49 (1994)1929; S. Hyun, G.H. Lee and J.H. Yee, Phys. Lett. B322 (1994)182; J. Chan and R. Mann, gr-qc/9612026.

[22] B. Breitenlohner and D.Z. Freedman, Phys. Lett. B115 (1982) 197; Ann. Phys. 114 (1982) 117.

[23] R. Emparan, hep-th/9804031.

[24] V. Balasubramanian, P. Kraus, and A. Lawrence, hep-th/9805171. 